\title{
Carnets
}

Revue électronique d'études françaises de l'APEF

Deuxième série - 14 | 2018

Études de génétique théâtrale et littéraire

\section{De L'Inventaire du planteur au Mousse et au Cabin boy : Réorientation et recadrage d'une histoire antiesclavagiste d'É. Souvestre en pièce de théâtre}

Barbara T. Cooper

\section{OpenEdition}

Journals

Édition électronique

URL : http://journals.openedition.org/carnets/8649

DOI : $10.4000 /$ carnets.8649

ISSN : 1646-7698

Éditeur

APEF

Référence électronique

Barbara T. Cooper, « De L'Inventaire du planteur au Mousse et au Cabin boy : Réorientation et recadrage d'une histoire antiesclavagiste d'É. Souvestre en pièce de théâtre », Carnets [En ligne], Deuxième série - 14 | 2018, mis en ligne le 30 novembre 2018, consulté le 02 mai 2019. URL : http:// journals.openedition.org/carnets/8649; DOI : 10.4000/carnets.8649

Ce document a été généré automatiquement le 2 mai 2019.

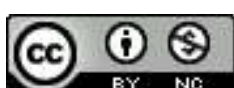

Carnets est mis à disposition selon les termes de la licence Creative Commons - Atribution - Pas d'utilisation commerciale 4.0 International. 


\title{
De L'Inventaire du planteur au Mousse et au Cabin boy : Réorientation et recadrage d'une histoire antiesclavagiste d'É. Souvestre en pièce de théâtre
}

\author{
Barbara T. Cooper
}

\begin{abstract}
Ah! s'il s'était agi de choisir, parmi les écrivains, un digne apôtre de l'idée populaire dans le sens du progrès moral qui élève l'humanité ; de choisir un homme de conviction et de pratique, éminemment honorable par son caractère et par ses œuvres tout ensemble, il y en avait un à désigner : c'était le regrettable [sic pour regretté] Émile Souvestre. (Muret, 1862 : I, 105-106).
\end{abstract}

L'Inventaire du planteur, nouvelle antiesclavagiste d'Émile Souvestre, est publiée en feuilleton dans le quotidien parisien Le National les 9,10 et 13 février 1838. À la fin de l'histoire, l'auteur précise la source dont il s'est inspiré pour rédiger son texte : « Le fond de ce récit est vrai et nous en avons emprunté l'idée à l'ouvrage si intéressant et si curieux de miss Martineau (...). [Son] livre sur la Société américaine renferme (...) des détails affreux sur l'esclavage des Noirs dans les états du sud »(Souvestre, 1839 : 275). Un an après, Henri de Saint-Georges et le compositeur Hippolyte Monpou transforment à leur tour le récit de Souvestre en opéra-comique (Le Planteur, Théâtre de l'OpéraComique, le $1^{\text {er }}$ mars 1839) - transformation que nous avons étudiée ailleurs ${ }^{1}$. Mais l'histoire ne s'arrête pas là. En 1846, Souvestre décide de reprendre sa nouvelle pour en tirer une comédie-vaudeville en deux actes intitulée Le Mousse qui est créée au Théâtre des Variétés le 18 janvier 1846 avec l'acteur Hugues Bouffé dans le rôle-titre². Comme on le verra, cette pièce, réorientée et recadrée en fonction de critères d'ordre générique, théâtral et culturel, se distingue tout à la fois de L'Inventaire du planteur et du Planteur. Le 
Mousse passe ensuite en Angleterre où il est représenté (Theatre Royal, Adelphi, le 9 mars 1846) et publié sous le titre The Cabin Boy par le dramaturge Edward Stirling qui omet toute mention du texte français qu'il reprend à son compte ${ }^{3}$. L'actrice Madame Céleste, à qui Stirling dédie son drame, incarne le personnage éponyme en travesti ${ }^{4}$. Nous nous proposons d'examiner ici des éléments de la génétique du Mousse et de sa traduction par Stirling afin de montrer à quel point la composition et l'évolution d'une œuvre s'inscrit dans la durée et dans des contextes multiples.

\section{L'Inventaire du planteur : suite et source}

2 Il faudrait sans doute commencer par rappeler l'intrigue de L'Inventaire du planteur, la nouvelle que Souvestre a écrite à partir d'une anecdote rapportée dans La Société américaine de Martineau. Dans cette nouvelle, Jenny Makensie est fille d'un planteur fort endetté de l'Alabama qui meurt peu après le début du récit en laissant la jeune femme face à ses nombreux créanciers. (Son fiancé est parti à Boston afin de gagner de l'argent et sera donc absent de l'histoire.) Le plus important des créanciers de Makensie s'appelle M. Jackson, riche propriétaire d'une plantation voisine. C'est un homme impitoyable et implacable que Jenny trouve effrayant. Jackson lui dit que si elle consent à l'épouser, il oubliera les dettes de Makensie. Elle refuse et ne peut espérer qu'en l'aide d'un oncle qui arrive du New Hampshire. On dresse l'inventaire du planteur défunt, on dépouille ses livres de compte et papiers et on invite les créanciers à se présenter. La solde des terres, de l'habitation, des récoltes et des esclaves de Makensie ne monte qu'à vingt mille dollars - somme insuffisante pour satisfaire les créanciers avides. C'est alors que Jackson découvre que la femme de Makensie, feue la mère de Jenny, était une esclave non affranchie. Selon la loi, Jenny est donc esclave aussi et fait partie des biens de son père à vendre. L'oncle n'a pas les moyens de racheter la liberté de Jenny, mise à l'encan avec les autres possessions de son père. C'est à Jackson qu'elle est adjugée. Pour échapper au cruel destin qui l'attend (la prostitution et la servitude), Jenny se jette par la fenêtre, se noyant dans le fleuve Alabama dès qu'on donne la quittance des dettes de son père à son oncle. Par cette conclusion, Souvestre condamne sans ambages l'esclavage et le capitalisme et laisse entendre que, pour se soustraire à ces deux systèmes intimement imbriqués, la seule solution possible quand on est pauvre et face à des gens sans cœur, c'est la mort. Pour tragique qu'elle soit, cette fin héroïse Jenny qui refuse de se résigner à un sort infamant et liberticide.

3 Il est évident que l'histoire de Souvestre, transformée en opéra-comique par SaintGeorges et Monpou, ne peut pas se terminer par une fin aussi sombre, même si Jenny Makensie se voit toujours redéfinie par la loi et les créanciers de son père comme esclave et bien meuble dans leur ouvrage ${ }^{5}$. Des conventions génériques s'y opposent formellement (Bara, 2003 : 229-240). Aussi, pour arriver à une conclusion plus heureuse, Saint-Georges modifie-t-il non seulement l'évolution de l'intrigue mais aussi le caractère des personnages qu'il hérite de Souvestre, auxquels il en ajoute d'autres de sa création ${ }^{6}$. Nous ne reviendrons pas sur cette vérité que nous avons déjà élucidée dans nos travaux précédents. Il sera bon, cependant, de se rappeler que la condamnation de l'esclavage et du capitalisme est atténuée dans l'opéra-comique par rapport à la nouvelle de Souvestre. Est-ce que le message abolitionniste et humanitaire de L'Inventaire sera également estompé quand Souvestre retravaille sa nouvelle en pièce de théâtre? Le critique dramatique de la Revue indépendante prétend que non? 
Cependant, quand Souvestre reprend son texte de 1838 en 1846 pour en faire une comédie-vaudeville, il doit, à l'instar de Saint-Georges, s'accommoder de nouvelles contraintes génériques. Tout comme un opéra-comique, une comédie-vaudeville doit se terminer heureusement. Et comme l'opéra-comique, la comédie-vaudeville mélange couplets chantés et répliques parlées, avec cette différence que les airs employés dans Le Mousse seront, à de rares exceptions près, empruntés à des œuvres déjà existantes et non pas composés spécialement pour le nouvel ouvrage - comme le veut les conventions du genre et les décrets gouvernant le répertoire des différents théâtres. Souvestre devra aussi tenir compte du contexte culturel qui, avec le passage du temps et la création du Planteur, entre autres, s'est modifié. Enfin, en écrivant le rôle-titre de sa pièce pour l'acteur Bouffé, il doit penser non seulement au répertoire et à l'emploi de ce comédien, mais aussi aux attentes du public qui fréquente le Théatre des Variétés où Le Mousse sera montée.

\section{Réorientation et recadrage}

Le titre de la pièce de Souvestre (Le Mousse), le décor ${ }^{8}$ et le chœur chanté par des matelots occupés à décharger un navire au lever du rideau, annoncent tout de suite la réorientation, le recadrage de L'Inventaire. On découvre d'entrée en jeu que l'action se passe non pas dans un des états à esclaves des États-Unis, mais aux Antilles. On comprend aussi que, s'il est toujours question de dettes, d'esclavage et de créanciers dans cette pièce, ce sont des matelots, et surtout le mousse éponyme, qui joueront un rôle prépondérant dans l'évolution de l'intrigue ${ }^{9}$. Par ailleurs, les timbres des couplets chantés en chœur par l'équipage au début et à la fin de la pièce de Souvestre sont empruntés de $L a$ Salamandre, comédie-vaudeville de Livry, Pittaud de Forges et Leuven (Théâtre du PalaisRoyal, 16 juin 1834) - œuvre inspirée par le roman maritime au même titre d'Eugène Sue ${ }^{10}$ . Or, les airs pour ces deux chœurs furent composés exprès pour La Salamandre par Hippolyte Monpou. Clin d'œil ou hommage indirect au compositeur du Planteur mort en $1841^{11}$ ? C'est en tout cas un élément génétique qu'il importe de constater puisque cet écho musical tisse un lien entre la pièce de Souvestre et d'autres œuvres appartenant à la littérature maritime - lien qui n'est point présent dans L'Inventaire du planteur ni dans Le Planteur, mais dont on trouve la trace dans de nombreuses pièces de l'époque ainsi que dans bon nombre de fictions narratives ${ }^{12}$.

6 L'incorporation d'éléments coloniaux et maritimes dans Le Mousse n'a d'ailleurs rien d'étonnant quand on pense aux origines bretonnes de Souvestre et à la publication, entre autres textes, de La Colonie (1841) ${ }^{13}$, nouvelle de Souvestre dont le critique Joël Cherbuliez écrit: «Il y a des détails pleins d'originalité dans le tableau de cette colonie, dont M. Souvestre a étudié les mœurs et l'histoire dans les ouvrages des auteurs contemporains " (Cherbuliez, $1841: 263$ ), et de Julien, nouvelle bretonne publiée en $1844^{14}$. Comme on le verra, c'est en combinant des éléments tirés de L'Inventaire du planteur avec d'autres appartenant à Julien que Souvestre créera Le Mousse ${ }^{15}$. Aucun critique de l'époque ne semble l'avoir remarqué. 


\section{Les sources génétiques du Mousse}

7 Pour bien saisir la manière dont procède Souvestre en composant Le Mousse, on peut penser à un jeu de cartes. Au cours du jeu on mêle les cartes, on les distribue et on décide de garder ou de rejeter les cartes qu'on tient en main. Ces opérations et d'autres créent à chaque fois une nouvelle donne. Or, c'est par un travail combinatoire semblable que Souvestre va créer Le Mousse à partir de L'Inventaire et Julien, entre autres textes. On trouve facilement des passages de dialogue et des péripéties dans Le Mousse qui sont empruntés avec peu ou pas de changements d'un de ses deux textes narratifs que Souvestre reprend pour créer sa pièce. Il y a, par exemple, la séquence qui s'étend de la page 156 jusqu'à la page 159 dans Julien (Souvestre, 1844). Là, le personnage éponyme, jeune pilotin breton, explique à un étranger l'histoire de ses liens avec M. de Laroche, propriétaire et armateur important à Roscanvel ${ }^{16}$, et sa fille, Mlle Blanche. Tout a commencé quand Julien et sa mère, pauvre et malade, habitaient une petite maison insalubre au bord de l'eau et n'avaient rien à manger. Découvrant le dénuement de cette famille lors d'une promenade, Blanche et son père l'ont secourue puis, à la mort de la mère, ils l'ont fait enterrer au cimetière et aidé Julien à s'embarquer comme novice au bord d'un navire. Dans Le Mousse, I, sc. 5, Julien raconte l'histoire des secours que lui et sa mère ont reçus de Mme Laroche et sa fille, Jenny, dans des circonstances analogues sinon identiques. Cependant, si le nom de la famille généreuse et secourable (Laroche) qui vient en aide à Julien et sa mère reste le même d'une œuvre à l'autre, le fond de l'histoire des Laroche a changé.

Cela s'explique par le fait que Souvestre insère, vers la fin de sa pièce, une séquence importante extraite de L'Inventaire du planteur. Les scènes 9 et 10 de l'acte II du Mousse reproduisent, à quelques différences près, la vente aux enchères de Jenny Makensie que Souvestre a racontée dans sa nouvelle de 1838 (Souvestre, 1839 : 263-275). Il y a même des répliques dans la pièce qui sont tirées sans changement de la nouvelle. Cet emprunt ne manque pas d'avoir des conséquences sur la présentation de Jenny tout au long du Mousse . Par exemple, le prénom Jenny vient de L'Inventaire. Garder le prénom Blanche, donné à Mlle Laroche dans Julien, aurait été impensable dans Le Mousse où il s'agit de faire découvrir l'ascendance africaine de la jeune femme et la faire vendre aux enchères ${ }^{17}$. Les dettes de Jenny et les menaces qui pèsent sur elle de la part de ses créanciers sont également repris de L'Inventair ${ }^{18}$. Le fait que la mère de Jenny, déjà morte avant le début de la pièce, fut esclave sans que Jenny le sache est un autre souvenir de L'Inventaire et est indispensable au revirement qui voit la jeune femme adjugée à $M$. Vincent, riche colon intraitable qui achète les créances des autres et enchérit sans cesse afin de prendre possession de Jenny. Mais Souvestre ne dénouera pas la crise dans Le Mousse de la même manière qu'il l'avait fait dans L'Inventaire.

9 Si les origines de Jenny sont découvertes, dans Le Mousse comme dans L'Inventaire, par moyen d'un document retrouvé lors de la vente des biens de sa famille, dans la pièce ce n'est pas le planteur cruel qui repère ce papier important. C'est Julien. Il part à la Dominique en plein orage chercher l'acte de naissance de Jenny et l'acte de mariage de sa mère - documents qui doivent protéger la jeune femme de la saisie en prouvant qu'elle est encore mineure et donc exemptée du remboursement de ses dettes ${ }^{19}$. L'acte de naissance que le jeune mousse recouvre non sans mal répond à ses souhaits. Mais c'est sans compter sur la révélation que renferme l'acte de mariage, acte que Julien rapporte 
sans l'examiner et que M. Vincent lira. Ce document prouve que Mme Laroche se nommait tout simplement Marthe et qu'elle fut l'esclave non affranchie de M. Laroche. Cette double séquence (orage et vente aux enchères de Jenny) s'accorde parfaitement avec les attentes du public du théâtre des Variétés non seulement parce qu'elle fait monter la tension émotionnelle dans la pièce, mais aussi parce qu'elle fournit l'occasion de spectacles sidérants.

Pour parer aux effets de l'achat de Jenny et amener la conclusion heureuse voulue par les conventions du genre (le mariage avec son cousin Henry), il faudrait ajouter un nouveau revirement à la pièce. Souvestre va puiser encore une fois dans Julien pour le créer. Dans la nouvelle bretonne, il est question d'un pirate, capitaine de l'Ariel, que l'oncle de Julien avait rencontré en mer, qu'il identifie comme le voltigeur hollandais de la légende et retrouve en la personne de $\mathrm{M}$. Achille, l'homme qui jette son dévolu sur Blanche Laroche (Souvestre, 1844: 167-168, 266-268). Cette histoire du Hollandais et de l'Ariel figure également dans Le Mousse (I, sc. $5^{20}$, et II, sc. 12-16) où elle fournit, comme dans la nouvelle de 1844 , le moyen de triompher du méchant qui craint de se voir dénoncé aux autorités comme flibustier. Dans la pièce, M. Vincent renoncera à Jenny et l'affranchira avant de quitter l'île. Elle pourra donc partir en France avec Henri, Julien et le reste de l'équipage ${ }^{21}$.

\section{L'acteur et son emploi - autres composants de la génétique théâtrale}

11 Pourquoi Souvestre reprend-il si souvent des éléments tirés de Julien, nouvelle sans visées abolitionnistes, alors que le but du Mousse est de dénoncer, comme il l'avait fait dans L'Inventaire, les méfaits de l'esclavage ? Si l'on songe que Souvestre écrit le rôle-titre de sa pièce pour Bouffé, on comprend mieux ce choix. Le personnage de Julien dans la nouvelle qui porte son nom s'aligne parfaitement sur le modèle des protagonistes typiquement joués par cet acteur alors que L'Inventaire n'offre aucun personnage important qui puisse lui convenir. Il suffit de lire l'éloge du comédien que le critique Eugène Faure (il signe Em. D.) publie dans La Revue indépendante pour comprendre le choix qui s'impose à Souvestre en cherchant un modèle pour le personnage éponyme du Mousse.

À tous ceux qui connaissent Bouffé, [la] narration des péripéties du second acte donnera facilement l'idée de tout ce que son talent a dû tirer de cette situation. C'est surtout ce second acte qui fait et assure le succès de la pièce. Là, en effet, le comédien sérieux renonce sans hésitation à toutes les coquetteries enfantines qui, pour beaucoup de gens, font partie intégrante des facultés de cet artiste excellent. Là, plus de couleur locale, mais ce qu'on voit en tout temps, en tout lieu, la faiblesse opprimée, la faiblesse qui pleure et qui crie, qui cherche et qui trouve l'éloquence attendrissante, et cependant ne peut toucher un cœur de roche ; la faiblesse repoussée qui, enfin, se relève et se grandit jusqu'au désespoir. Brusques transitions, accents pleins de larmes, éloquence du geste et du regard, amère et noble raillerie du bon droit sauvé, Bouffé, dans ce rôle, a trouvé tout ce qui lui faut, et il a tout rendu avec cette vive simplicité d'observation et ces élans du cœur que vous savez. (...) Somme toute, le Mousse ajoute un bon rôle au répertoire de Bouffé (Faure $1846: 262,263$ ).

Son collègue de L'Union des arts abonde dans le même sens.

Le Mousse, vaudeville en deux actes de M. É. Souvestre, est un petit bonhomme leste et pimpant, sensible et courageux qui compromet, sauve, recompromet et ressauve Jenny sa bienfaitrice, jusqu'à ce que définitivement, il fasse son bonheur.

Bouffé est charmant de gaîté, d'espièglerie et de sensibilité dans ce rôle qui 
marquera parmi ses plus belles créations. C'est ce qu'on peut appeler un succès

d'acteur (Guéroult, 1846 : 31, souligné dans l'original) ${ }^{22}$. Edward Stirling s'accapare-t-il très vite du Mousse et le modifie-t-il. The Cabin Boy traduction exacte du titre de Souvestre - est présenté alors au public anglais comme une œuvre originale et la nouvelle pièce est créée le 9 mars 1846 au Theatre Royal, Adelphi avec Mme Céline Céleste, actrice et gérante de ce théâtre, dans le rôle-titre. (Les dramaturges anglais ont l'habitude de reprendre à leur compte des textes étrangers et on ne songe pas souvent à condamner la pratique, même quand on signale la source de qui n'en sont pas moins esclaves, parce que leurs bisaïeules étaient négresses. Mme Morand. Oui, monsieur, ce qui fait les noirs, ce n'est pas la couleur, c'est l'origine. Balandier. Ah ! très bien; voilà pourquoi cette jeune fille [femme qu'il a rencontrée en ville] est une femme de couleur, tandis que madame Morand est une femme blanche. Mme Morand. Oui, monsieur ! Balandier. C'est à confondre, madame, c'est à confondre! (Souvestre 1846: 20 ; emphase dans l'original).

sociales, politiques et commerciales qui s'y rattachent dans les colonies, mais aussi de préparer la découverte, plus loin, des origines «noires » de Jenny et du statut d'esclave qu'elle hérite de sa mère ${ }^{25}$. La comédie engendrée par la méprise de Balandier ici se transformera alors en situation dont les conséquences sont bien plus graves.

\section{Du Mousse au Cabin Boy - des conventions et contextes différents}

La nouvelle du succès de la pièce de Souvestre ne tarde pas à traverser la Manche. Aussi 
l'œuvre ${ }^{26}$.) Dans quelle mesure la pièce de Stirling, calquée sur Le Mousse de Souvestre, diffère-t-elle de son modèle et comment expliquer les différences entre les deux œuvres ?

The Cabin Boy n'est ni une traduction exacte ni une reproduction intégrale du Mousse même s'il reprend l'essentiel de la pièce de Souvestre, comme l'atteste le résumé de l'intrigue publié dans The Times (Anon., 1846: 6). Cela s'explique par des différences dans le contexte culturel et théâtral des deux côtés de la Manche et les attentes divergentes des publics londoniens et parisiens. Qui plus est, en arrivant sur le sol anglais Le Mousse est coupé de ses origines (l'œuvre et les convictions humanitaires de Souvestre) et est rattaché au corpus dramatique de Stirling dont les pièces sont citées sur la page-titre de la brochure du Cabin Boy. Le Mousse est également dégagé des conventions génériques et dramatiques qui ont dictée sa composition en France. The Cabin Boy n'est pas une comédie-vaudeville où des couplets chantés sur des airs connus occupent une place importante. Il est désigné «a drama » et les quelques passages de musique orchestrale qu'on y trouve sont joués pour souligner une émotion ou accentuer un tableau dramatique ${ }^{27}$. Il ressemble donc à ce qu'on appellerait un mélodrame en France et de ce fait correspond au répertoire de l'Adelphi.

Si The Cabin Boy, comme Le Mousse, se rattache par ses personnages à la littérature maritime, cette littérature diffère de part et d'autre de la Manche, les Anglais y abordant plus fréquemment que les Français des questions de nationalisme et d'impérialisme (Bratton, 1991 ; Davies, 1988), d'hiérarchie maritime et sociale (Carlson, 1996 ; Cox, 1996 ; Burroughs, 2011) ou du travail des enfants (Lawrence, 2008) ${ }^{28}$. La pièce de Souvestre, sans être fait sur le modèle anglais du mélodrame nautique, renferme néanmoins des éléments que l'on y retrouve souvent, comme la flagellation des marins (Julien est constamment menacé de coups de garcette par son oncle Lagarcette) et de rivalités amoureuses ou morales entre un nanti de pouvoir et d'argent ou un pirate et un marin dont le rang fait de lui un inférieur. Dans les années 1820-1830 l'Adelphi fait jouer bon nombre de pièces à sujet maritime dont The Flying Dutchman, or the Phantom Ship (Fitzball, 1826), The Pilot, a Nautical Burletta, du même auteur (1825) et The Blue Jackets, or Her Majesty's Service de Stirling lui-même $(1838)^{29}$. On ne s'étonnera donc pas de voir la pièce française adaptée et mise à l'affiche de ce théâtre. Mme Céleste joue Julien en travesti et le frontispice de la brochure du Cabin Boy la montre visant M. Vincent d'un fusil, le menaçant de mort. C'est un des moments les plus héroïques de la pièce - avec le départ du jeune mousse en plein orage - et met en valeur ses talents d'actrice et de gérante de théâtre soucieuse de la mise en scène (Moody, 1999 : 114-119).

Si la pièce se déroule globalement comme son modèle français, il y a néanmoins des différences entre les deux œuvres. Certains passages ou scènes sont supprimés, d'autres modifiés ou ajoutés. Il serait impossible de détailler ici tous les changements effectués par Stirling. En nous limitant à quelques exemples portant sur les questions de l'esclavage et de race, nous croyons toucher à un aspect essentiel de la transformation que subit l'œuvre de Souvestre. Dans Le Mousse, Balandier parle de la façon dont il traitera les Noirs qu'il pense acheter en s'installant comme planteur à la Guadeloupe. Julien lui fait savoir que s'il maltraite ses esclaves, ils pourront décider de retourner en Afrique. Aussi, précise-t-il, pour " voyager sans passeport » ni l'autorisation de leur maître, les esclaves ont-ils l'habitude de se pendre. Ils pourront même décider d'amener leur maitre avec eux en le pendant à leurs côtés (I, sc. 11), ce qui fait réfléchir Balandier. Cette histoire rappelle une anecdote qui circule depuis le XviII siècle et que l'on retrouve chez Granier de Cassagnac (1843: 159), entre autres. Si elle signale des croyances (supposées ?) caribo- 
africaines, elle révèle en même temps les malheurs et abus auxquels les esclaves sont sujets et renforce l'image négative que Souvestre trace de l'esclavage dans sa pièce. Cette conversation, dont le but est, aussi, de se moquer de Balandier, est absente du Cabin Boy.

Maître de danse ridicule chez Souvestre, Balandier devient vendeur de "curiosités naturelles » ridicule dans la pièce de Stirling. Le dramaturge anglais profite de ce changement de profession pour modifier un développement déjà présent dans l'œuvre de Souvestre. Dans Le Mousse, lors de la vente aux enchères, Balandier fait l'acquisition, pour la somme de 2000 francs, de « cinq cochons, vingt-trois poules et un âne » et les planteurs se moquent de lui (II, sc. 2). Dans le Cabin Boy, il devient propriétaire d'une femme voilée qui, à ce qu'il croit, est une jeune et belle esclave blanche qu'il entend ramener à Paris pour l'exhiber comme curiosité. Les planteurs se moquent de lui et Balandier finit par découvrir qu'il a acheté une vieille Négresse fort laide qui baragouine un anglais " petit nègre » et qui courra après lui pour l'embrasser (Stirling, 1846:17-18). Hazel Waters a raison de voir dans cette séquence une représentation grotesque de la lascivité souvent attribuée aux femmes noires et de comparer cette première vente marquée par la dérision à celle de Jenny plus loin où c'est le pathos qui règne. Dans les deux cas, une femme noire est traitée de marchandise et est vendue, mais la femme à peau foncée est raillée - comme Balandier - alors que celle qui a une peau claire suscite la commisération et la pitié des «bons» marins et planteurs ainsi que des spectateurs (Waters, 2007 : 121-122 et 138-139). Aussi, contrairement à ce qui se passe dans Le Mousse, l'esclavage et la commodification des êtres humains ne semblent-ils pas être condamnés dans cette scène du Cabin Boy. C'est surtout le caractère de l'acheteur individuel (ridicule ou traitre) et les caractéristiques des femmes vendues (noire, laide et lubrique ou blanche, belle et bonne) qui sont soulignés sans que les institutions et idéologies (colonialisme, capitalisme, racisme) qui sous-tendent et « justifient » de telles pratiques soient dénoncées.

\section{Conclusion}

Comme nous espérons l'avoir montré ici et dans nos travaux précédents, l'anecdote abolitionniste rapportée par Harriet Martineau dans De la société américaine a engendré plusieurs œuvres littéraires entre 1838 et 1846. Fictionnalisée et transformée par Souvestre en nouvelle antiesclavagiste (L'Inventaire du planteur), puis métamorphosée en opéra-comique par Saint-Georges et Monpou (Le Planteur) avant de devenir une comédievaudeville sous la plume de Souvestre (Le Mousse) et un drame chez Stirling (The Cabin Boy ), l'histoire d'une jeune femme qui apprend son ascendance noire et son statut d'esclave évolue selon les normes génériques et des contextes nationaux, esthétiques et politiques en perpétuel devenir. En cours de route, Jenny perd de plus en plus d'autonomie et de détermination. Courageuse et décidée à sacrifier sa vie pour échapper à l'esclavage dans L'Inventaire du planteur, dans Le Planteur elle finit par épouser un homme généreux et bon par reconnaissance. Dans Le Mousse et dans The Cabin Boy, Jenny est encore plus passive et, sans le courage de Julien, elle resterait au pouvoir du traître. Étudier la génétique des textes de Souvestre et Stirling fait ressortir les similarités et les différences entre leurs ouvrages. Plus généralement, cela permet de revoir la notion d'une histoire littéraire exclusivement nationale et de réexaminer notre conception de l'originalité artistique. Nos analyses ne peuvent qu'y gagner. 


\section{BIBLIOGRAPHIE}

ANON (1846). « Premières Représentations », L'Argus, 6 août, pp. 1-2.

ANON (1846). «Adelphi Theatre », The Times [Londres], 10 mars, p. 6.

ANON (1840). « Théâtre anglais », Le Ménestrel, 14 juin, pp. 2-3.

ANON (1839). «Théâtre de l'Opéra-Comique. Première Représentation du Planteur », Le Courrier

français, no 63, 4 mars, p. 3, feuilleton.

ARTHUR, Stéphane (2016). « Se donner la mort sur la scène romantique. Enjeux esthétiques, sociaux, politiques », European Drama and Performance Studies, t. 2, n 7, pp. 145-156.

BARA, Olivier (2003). « D'un corpus hybride : l'opéra-comique, au carrefour des genres dramatiques et lyriques », Lieux Littéraires / La Revue, n 7-8, pp. 229-240.

BAYARD, Jean-François et de BIÉVILLE [Charles-Henri-Étienne-Edmond Desnoyers de Biéville] (1840). Les Enfants de la troupe. Paris : Marchant.

BAYARD, Jean-François et Émile VANDERBURCH (1836). Le Gamin de Paris. Paris : Marchant.

BOUFFÉ, Hugues (1880). Mes Souvenirs, 1800-1880. Paris : Dentu.

BRATTON, J. S. (1991). « British Heroism and the Structure of Melodrama » in Acts of Supremacy:

The British Empire and the Stage, 1790-1930. Manchester: Manchester UP, pp. 18-61.

BURROUGHS, Robert (2011). « Sailors and Slaves : The 'Poor Enslaved Tar' in Naval Reform and Nautical Melodrama », Journal of Victorian Culture, t. 16, no 3, pp. 305-322.

C***, Delmare [Théodore de BANVILLE ?] (1846). « Théâtres », Le Tintamarre, 25 jan., p. 5-6. CARLSON, Marvin (1996). « He Never Should Bow Down to a Domineering Frown : Class Tensions and Nautical Melodrama » in Michael Hays et Anastasia Nikolopoulou, eds. Melodrama: The Cultural Emergence of a Genre. New York : St. Martin's Press, pp. 147-166.

CARMOUCHE, Pierre-Frédéric-Adolphe et Frédéric de COURCy (1839). La Chaste Suzanne, opéra ; musique de Monpou. Paris : Mifliez ; Tresse.

CHOQUET, Victor (1851). « Théâtre des Folies-Dramatiques », Le Daguerréotype théâtral, 30 jan., p. 2. COOPER, Barbara T. (2019). « Le Planteur, d'Henri de Saint-Georges et Hippolyte Monpou (1839) : Un opéra-comique aux antécédents multiples et transmodaux » in Ana Clara Santos, Sophie Lucet (éds.), Parcours de génétique théâtrale : brouillon(s), (ré)écriture(s) et transmodalisation(s) dramatique(s). Rennes : PUR (sous presse).

CORBIÈRE, Édouard (1837). « Des emprunts libres faits par notre époque à la littérature maritime », La France Maritime, t. 3, pp. 82-83.

cox, Jeffrey N. (1996). « The Ideological Tack of Nautical Melodrama » in Michael Hays et Anastasia Nikolopoulou (éds.). Melodrama : The Cultural Emergence of a Genre. New York : St. Martin's Press, pp. 167-189.

D., Em. [pseud., Eugène FAURE] (1846). «Revue théâtrale. Théâtre des Variétés », La Revue indépendante, 25 jan., pp. 261-263. 
D., J. (1846). « On Pieces Adapted From the French », The Theatrical Times, 24 oct., pp. 165-166. DAVIES, Jim (1988). « British Bravery, or Tars Triumphant : Images of the British Navy in Nautical Melodrama », New Theatre Quarterly, t. 4, no 14, pp. 123-143.

DESHAYES, L. (1853). « Progrès et popularité de la marine, de 1834 à 1848 », La France maritime, t. 3, pp. 1-4.

FITZBALL, Edward (1826). The Flying Dutchman, or the Phantom Ship, a Nautical Drama. London : Lacy. [Adelphi Theatre].

FITZBALL, Edward (1825). The Pilot, A Nautical Burletta. London : John Cumberland. [Adelphi Theatre].

FOUQUEAU DE PUSSY, Mme J. J. (1846). « Revue des théâtres. Le Mousse », Le Journal des Demoiselles, mars, pp. 83-90. (Voir Google Books).

FOURNIER, Narcisse (1835). Le Menuet de la reine. Paris : Marchant.

GAUTIER, Théophile (1846). « Théâtres. Variétés », La Presse, 19 jan., pp. 1-2, feuilleton.

GRANIER DE CASSAGNAC, Adolphe (1843). Voyages aux Antilles. Les Antilles françaises. Paris : Au comptoir des imprimeurs réunis.

GRÉGOIRE, Achille (1830). Bal champêtre au cinquième étage, ou Rigolard chez lui. Paris : Paul Banes. GUÉROULT, C[onstant] (1846). « Théâtres ", L’Union des arts, fév., pp. 30-32.

LAWRENCE, David Haldane (2008). « Performing Working Boys: the Representation of Child Labour on the Pre- and Early Victorian Stage ", New Theatre Quarterly, t. 24, n² 2, pp. 126-140.

HOWARD, Edward. (1841). « Le Matelot anglais », in Les Anglais peints par eux-mêmes. Tr. Émile de Labédollierre. Paris : Curmer, t. 2, pp. 326-335.

HAILL, Catherine. http://www.elta-project.org/theme-nautical.html consulté le 19 juin 2018.

JAL, Augustin (1833). Scènes de la vie maritime. Paris : Ch. Gosselin, t. 2, pp. 103-115.

LECOMTE, Jules (1837). «Traditions et superstitions maritimes. Le Grand Voltigeur hollandais », La France maritime, t. 2, pp. 27-29.

LIVRY, Charles de, Philippe-Auguste-Alf. PITTAUD DE FORGES et Adolphe de LEUVEN (1834). La Salamandre. Paris : Barba, Quoy, Bezou.

MARC-MiChel et Émile FONTAINE (1840). Rifolard, épisode d'une vie agitée. Paris : Henriot.

MARTINEAU, Harriet (1838). De la société américaine. Tr. Benjamin Laroche. Paris : Charpentier.

MÉLESVILLE (Anne-Honoré-Joseph Duveyrier, dit), et Jean-François-Alf. BAYARD (1844). Le Chevalier de Grignon. Paris : imp. de Lacombe.

MOODY, Jane (1999). « Women and Playwriting in Nineteenth-Century Britain » in Tracy C. Davis, Ellen Donkin, eds., Illusions of Authorship. Cambridge ; New York : Cambridge UP, pp. 99-124.

MURET, Théodore (1862). Souvenirs et causeries d'un journaliste. Paris : Garnier frères, t. 1, pp. 105-106.

R. (1846) : « Théâtres », Le Constitutionnel, 19 jan., p. 1, feuilleton.

RICHER, Paul (s. d.). Diana, ou La Créole. Pièce inédite. (Théâtre des Folies-Dramatiques, 22 jan. 1851.) 
SAINT-GEORGES, Henri de et Hippolyte MONPOU $(1839$; 2015). Le Planteur. Paris : Barba ; réédition par Barbara T. Cooper, Paris : L'Harmattan (coll. « Autrement Mêmes »).

SOUVESTRE, Émile (1841a). Une Colonie, La Revue de Paris, t. 26 (7, 14, 21, 28 fév.), pp. 5-24, 92-115, 145-169, 228-253 et t. 27 (7 mars), pp. 24-50. (Voir aussi SOUVESTRE, 1841b.)

SOUVESTRE, Émile (1838). L'Inventaire du planteur, Le National, 9, 10 et 13 fév., pp. 1-3, feuilleton. SOUVESTRE, Émile (1839). L'Inventaire du planteur in Le Journaliste. Paris : Charpentier, t. 2, pp. 221-275.

SOUVESTRE, Émile (1844). Julien, La Revue de Paris, t. 28, (21 et 28 avr.), p. 153-181 et 247-268. SOUVESTRE, Émile (1846). Le Mousse. Paris : Tresse (« La France dramatique du dix-neuvième siècle »).

SOUVESTRE, Émile (1841b). Pierre et Jean. Paris : H. Souverain. (Jean Plébeau est le nouveau titre donné à Une Colonie quand cette nouvelle est reprise ici pour former le t. 19 des Euvres d'Émile Souvestre).

SOUVESTRE, Émile (1853). Récits et souvenirs. Paris : D. Giraud. (Julien y est publié, pp. 1-90). STIRLING, Edward (1838). The Blue Jackets, or Her Majesty's Service. London: Duncombe. [Adelphi Theatre]

STIRLING, Edward (1846). The Cabin Boy. London: Nassau Steam Press. [Adelphi Theatre].

SUE, Eugène (1832). La Salamandre. Paris : Renduel.

VAUCLARE, Théodore (1839). « Théâtres de Paris. Premières Représentations », Le Monde dramatique , sér. 2, t. 1, p. 140.

WATERS, Hazel (2007). Racism on the Victorian Stage: Representation of Slavery and the Black Character. Cambridge : Cambridge UP.

Y., Y. (1836). « Théâtres de Londres. $4^{\mathrm{e}}$ article » Le Constitutionnel, $1^{\mathrm{er}}$ jan., pp. 1-2 feuilleton.

\section{NOTES}

1. Voir Cooper, 2019 et la réédition de cet opéra-comique, Saint-Georges : 2015.

2. Souvestre, 1846 .

3. Stirling, 1846.

4. Une autre traduction anglaise, de Thomas Archer, The Cabin Boy or, The White Slave, s'annonce comme adaptation (« adapted from Le Mousse by Souvestre ») mais n'est ni publiée ni jouée. Le manuscrit fait partie des pièces soumises au Lord Chamberlain en avril-mai 1846. Voir La British Library, Plays Submitted to the Lord Chamberlain, Catalogue (Add. Mss.) No 42993 (11), ff. 286-371, vol. CXXIX. Je tiens à remercier Mme Jean Putnam du service des prêts interbibliothèques de l'Univ. du New Hampshire qui a obtenu une copie de ce manuscrit pour moi.

5. Si le suicide n'est pas exclu du théâtre français de cette époque, il est cantonné dans des drames, mélodrames et autres genres sérieux. Voir, entre autres, Arthur, 2016 : 145-156. Un bien meuble - personne, animal ou chose - peut être déplacé.

6. Vauclare, $1839: 140$, affirme que « Le planteur de M. Souvestre est un homme dur, implacable, despote, qui force par ses traitements une jeune esclave à se donner la mort; celui de M. de Saint-Georges, au contraire, est timide, bienfaisant, et sacrifie son bonheur au bien-être de son esclave ». Le personnage du fiancé de Jenny subit aussi une forte transformation dans l'opéra-comique, devenant un dandy endetté et volage. Le critique du Courrier français parle de « [...] la création de quelques physionomies, de celle de Caton, par exemple, dont la nouveauté est originale et comique ». Anon., $1839: 3$, feuilleton. Il faudrait mentionner aussi miss Arabelle, danseuse anglaise qui est la maîtresse du fiancé de Jenny et qui enlève le jeune homme à la fin de l'œuvre. 
7. "Ce petit ouvrage est (...) semé de plusieurs bons détails et de quelques généreux aperçus finement dessinés sur les honnêtes [mot sans doute ironique ici] spéculations du créancier, la vente des portraits de famille, la noblesse d'épiderme et ces obscures misères du pauvre, pour lesquelles M. Souvestre a toujours une bonne et douce larme ». Em. D., $1846: 263$. Voir aussi Anon., $1846: 1:$ « La littérature tourne au négrophile, et après le Mousse, de M. Émile Souvestre, MM. Anicet Bourgeois et Dumanoir [auteurs du Docteur Noir] ont voulu rompre une lance en faveur de l'émancipation des esclaves".

8. La scène se passe à la Guadeloupe sur une plantation au bord de la mer; une partie de l'arrière d'un navire et un canot amarré au quai sont visibles au lever du rideau.

9. Bouffé, $1880: 236$, affirme que « les choristes paraissaient d'un bout à l'autre » de cette pièce.

10. Livry, et. al., 1834. Sue, 1832. Plus loin dans Le Mousse (II, sc. 6, p. 24), c'est un quadrille emprunté à La Chaste Suzanne, de Carmouche et de Courcy, 1839, sur une musique de Monpou, qui sera repris.

11. Diana, ou La Créole, pièce inédite de Richer (Théâtre des Folies-Dramatiques, 22 jan. 1851), reprend le texte du Planteur mais se sert d'airs empruntés d'autres œuvres plutôt que de la partition de Monpou. Voir Choquet, $1851: 2$. oi d'éléments tirés de Julien dans Le Mousse que cette nouvelle n'est recueillie en volume qu'en 1853 ?

Fouqueau de Pussy, 1846 : 83-90 donne un résumé très détaillé de la pièce, ce qui nous dispense d'en faire autant. (On trouvera Le Journal des demoiselles sur Google Books.) Voir aussi Gautier

12. Voir Corbière, $1837: 82$ et Deshayes, $1853: 1-4$

13. L'action se passe à la Guadeloupe et on y rencontre un personnage, ancien acteur vivant sur les mornes, dont la conversation est semée de répliques de théâtre en vers. Souvestre, 1841a.

14. Est-ce pour empêcher les critiques de constater le réemploi d'éléments tirés de Julien dans Le Mousse que cette nouvelle n'est recueillie en volume qu'en 1853 ?

15. Fouqueau de Pussy, $1846: 83-90$ donne un résumé très détaillé de la pièce, ce qui nous dispense d'en faire autant. (On trouvera Le Journal des demoiselles sur Google Books.) Voir aussi Gautier, $1846: 1-2$, feuilleton.

16. Petit port dans la rade de Brest. Hormis quelques récits rétrospectifs, l'action se passe aux environs de Brest.

17. Voir les remarques infra tirées du Mousse sur les distinctions entre les races dans les colonies. Le nom et le caractère de Julien restent identiques dans les deux textes. Le fiancé de Jenny s'appelle Henri/Henry dans la pièce comme dans la nouvelle. D'autres noms tirés de Julien sont également repris dans la pièce.

18. La description que Vincent donne de lui-même (I, sc. 8) et que Julien donne de lui (I, sc. 10) fait écho à celle qu'on trouve de Jackson dans L'Inventaire (Souvestre $1839: 226$ ).

19. L'orage et le retour de Julien à la Guadeloupe alors qu'on l'imagine noyé n'ont pas d'équivalent dans L'Inventaire, mais se trouve bien dans Julien où il est question de recouvrer des documents qui compromettent la réputation de M. de Laroche (Souvestre, $1844: 178-181$ et 246-250).

20. Le récit de la légende est doté d'une belle mise en scène dans la pièce qui est absente de la nouvelle. Dans Le Mousse, les marins rassemblés pour manger et boire après leurs travaux disent « crik ! crak! ", interjection par laquelle ils demandent qu'on entame l'histoire qu'on a promis de leur raconter. Sur la légende du voltigeur hollandais, voir Lecomte, $1837: 27-29$. Voir aussi Jal, $1833: 112-115$ qui suggère que la légende de ce vaisseau fantôme aurait des liens avec la traite des Noirs et publie un poème inédit d'H. de Latouche qui va dans ce sens.

21. Delmare $C^{* * *}$, critique au Tintamarre, voit une similarité entre cette fin et celle du Marché de Saint-Pierre, pièce d'Antier et de Comberousse (1839) qu'il appelle L'Épave. ( $C^{* * *}, 1846: 5$.)

22. Voir aussi R., 1846: 1 feuilleton : « Bouffé, nous l'avons dit, joue le rôle de Julien ; il y a obtenu un très vif succès, surtout au dénouement, à l'instant où, prenant le corsaire en flagrant délit, et le traitant comme une cible du tir Lepage, il s'est mis à l'ajuster ainsi qu'une poupée. Dans cette scène très forte, très émouvante, qui est à vrai dire toute la pièce et fera tout son succès, M. Bouffé s'est élevé à de grands effets dramatiques (...). Une immense moisson de bravos ; voilà ce que M. Bouffé a récolté pour cette nouvelle étude consciencieuse et pour tant d'efforts pleins d'agitation et de pathétique ".

23. Voir, par exemple, Grégoire, 1830 où Bouffé joue un maître de danse ; Fournier, 1843 où il incarne le maître de danse Carlo Vestris ; et Mélesville et Bayard, 1844 où il joue Nogent, valet de chambre appelé le Chevalier de Grignon dans le monde où il donne des leçons de danse pour faire vivre son maître, aristocrate désargenté.

24. Dans une autre réminiscence du Planteur, dans Le Mousse Jenny ira se ranger parmi ses esclaves dès qu'on lui apprend son statut servile (II, sc. 9).

25. Cette conversation sert en même temps à expliquer la blancheur de peau de l'actrice qui incarne une femme de couleur sans se grimer.

26. Voir pourtant D., 1846:165-166. Voir aussi Y., $1836: 1$-2, feuilleton et ces remarques publiées dans Le Ménestrel en 1840. «Mais ces Messieurs [les dramaturges anglais] jugèrent inutile de mettre le public anglais dans la confidence de la stérilité de leur cerveau; ils changèrent 
presque toujours le titre des pièces qu'ils traduisirent, et, avec une impudente effronterie, les présentèrent comme les enfants de leur génie ». (Anon, $1840: 2$.)

27. On trouve une seule chanson dans le Cabin Boy (Stirling, $1846: 10$ ). Notons aussi que, conformément aux habitudes d'outre-Manche, les actes ne sont pas divisés en scènes dans la pièce anglaise.

28. Voir aussi Howard, 1841 : 326-335 pour une idée de l'image que les Anglais se font de leurs matelots.

29. Voir Haill, http://www.elta-project.org/theme-nautical.html sur les pièces à sujet nautique.

\section{RÉSUMÉS}

Une anecdote abolitionniste rapportée par Harriet Martineau dans De la société américaine a engendré plusieurs œuvres littéraires entre 1838 et 1846. Fictionnalisée et transformée par Souvestre en nouvelle antiesclavagiste (L'Inventaire du planteur), puis métamorphosée en opéracomique par Saint-Georges et Monpou (Le Planteur) avant de devenir une comédie-vaudeville sous la plume de Souvestre (Le Mousse) et un drame chez Stirling (The Cabin Boy), l'histoire d'une jeune femme qui apprend son ascendance noire et son statut d'esclave évolue selon des normes génériques et des contextes nationaux, esthétiques et politiques en perpétuel devenir. Étudier la génétique des textes de Souvestre et Stirling fait ressortir les similarités et les différences entre leurs ouvrages. Plus généralement, cela permet de revoir la notion d'une histoire littéraire exclusivement nationale et de réexaminer notre conception de l'originalité artistique. Nos analyses ne peuvent qu'y gagner.

An abolitionist anecdote recounted by Harriet Martineau in Society in America gave rise to several literary works written between 1838 and 1846. Transformed into an antislavery tale by Souvestre (L'Inventaire du planteur), then changed into a comic opera by Saint-Georges and Monpou (Le Planteur) before becoming a comédie-vaudeville written by Souvestre (Le Mousse) and a drama by Stirling (The Cabin Boy), the story of a young woman who learns she is of African descent and considered a slave evolves according to generic norms and national, aesthetic and political contexts that are constantly changing. Study of the genetics of Souvestre's and Stirling's texts brings out the similarities and differences between them. More generally speaking, it also allows for reconsideration of the notion that literary history in exclusively national in character and for reexamination of our concept of artistic originality. Our analyses clearly stand to gain from investigations such as this.

INDEX

Keywords : Theatrical genetics, Souvestre (Émile), Le Mousse, Cabin Boy, slavery

Mots-clés : Genèse théâtrale, Souvestre (Émile), Le Mousse, Cabin Boy, esclavage

\section{AUTEUR}

BARBARA T. COOPER

Université du New Hampshire (USA)

2btcooper[at]gmail.com 